\title{
THE CUT ELIMINATION THEOREM IN THE UNARY SECOND ORDER LANGUAGE
}

\section{MITSURU YASUHARA}

In $[\mathrm{T}]$, Takeuti has conjectured that the cut elimination theorem holds for the simple theory of types cast in the sequent calculus. This conjecture is true for the first order language, as Gentzen had shown in [G]. (Indeed, the conjecture was made after Gentzen had proved his "Hauptsatz.") The purpose of this Note is to show that the conjecture is true for the unary second order language $U$ (Theorem II). Our proof is based on another result of the Note which concerns the unary second order language with equality $U^{+}$. This theorem shows that the requirement of "predicativity" is not really a restriction on deducibility in $U^{+}$. In showing this, an essential use is made of the decision procedure for this language (cf. [A], for instance). The Note ends with a Remark in which the negative answer is given to a few possible extensions of above results. The fact that predicativity is an essential restriction on deducibility in $U$ seems to deserve mentioning here.

The primitive symbols of $U$ consist of free and of bound variables for elements, for propositions, and for sets, and symbols for negation, for disjunction, and for existential quantification. These symbols will be denoted by $a, b, \cdots, x, y, \cdots ; P, Q, \cdots, S, T, \cdots ; A()$, $B(), \cdots, X(), Y(), \cdots ; \neg, v$, and $\exists$, respectively. Symbols for other logical connectives may be used as abbreviations. The language $U^{+}$is richer than $U$ in that the symbol for equality, ${ }^{*}={ }^{*}$, is included. The well formed formulae and the sequents are defined in the standard way. We use German capitals and Greek capitals to denote formulae and finite sequences of formulae, respectively. A well formed formula is naturally called of first order if no bound variables for propositions or for sets occur in it. The sequents $\mathfrak{A} \rightarrow \mathfrak{A}$ are taken as the axioms of $U$, for all primitive formulae $\mathfrak{A}$. The rules of $U$ which are not found in [G] are those about variables for propositions and for sets. They are as follows:

$$
\exists p \rightarrow \frac{\mathfrak{A}(P), \Gamma \rightarrow \Delta}{\exists S \mathfrak{U}(S), \Gamma \rightarrow \Delta}
$$

with the provision that $P$ does not occur in the lower sequent;

Received by the editors March 12, 1965. 


$$
\rightarrow \exists p \frac{\Gamma \rightarrow \Delta, \mathfrak{A}(\mathfrak{B})}{\Gamma \rightarrow \Delta, \exists S \mathfrak{R}(S)}
$$

$\mathfrak{P}(\mathfrak{B})$ is the result of substitution ${ }^{1}$ of $\mathfrak{B}$ for $S$ in $\mathfrak{A}(S)$;

$$
\exists s \rightarrow \frac{\mathfrak{A}(A), \Gamma \rightarrow \Delta}{\exists X \mathfrak{U}(X), \Gamma \rightarrow \Delta}
$$

with the provision that $A$ does not occur in the lower sequent;

$$
\rightarrow \exists s \frac{\Gamma \rightarrow \Delta, \mathfrak{A}(\mathfrak{B})}{\Gamma \rightarrow \Delta, \exists X \mathfrak{U}(X)}
$$

$\mathfrak{Y}(\mathfrak{B})$ is the result of substitution of $\mathfrak{B}$ for $A$ in $\mathfrak{A}(A)$.

A sequent is called predicatively deducible, or pr-deducible, if it is deducible with the condition that the $\mathfrak{B}$ in $\rightarrow \exists p$ and $\rightarrow \exists s$ are of first order.

The deduction system of $U^{+}$is obtained from that of $U$ by adding the axiom sequents $\rightarrow a=a$ and $a=b, \mathfrak{U}(a) \rightarrow \mathfrak{U}(b)$ where $\mathfrak{A}(a)$ is a primitive formula.

Formulae $\mathfrak{A}$ and $\mathfrak{B}$ of $U^{+}$are called equivalent, in symbols $\mathrm{Eq}(\mathfrak{A}, \mathfrak{B})$, if both $\mathfrak{A} \rightarrow \mathfrak{B}$ and $\mathfrak{B} \rightarrow \mathfrak{A}$ are pr-deducible.

Leмма. Each formula of $U^{+}$is equivalent to a first order formula.

Proof. The notion of "Eq" is easily seen to be a congruence relation on the set of formulae. ${ }^{2}$ Hence, it suffices to show the lemma under assumptions that the given formula has only one occurrence of quantifier on a proposition or on a set variable, and that this quantifier is at the beginning of the formula. When the first quantifier is on a proposition variable and the formula under consideration is $\exists S \mathfrak{A}(S)$, the formula $\mathfrak{A}(P \vee \neg P) \vee \mathfrak{X}(P \& \rightarrow P)$ is easily shown to be an equivalent formula. In case the first quantifier is on a set variable, we can assume that the given formula is of the form

$$
\begin{array}{r}
\exists X \forall x\left[X\left(a_{1}\right) \& \cdots \& X\left(a_{m}\right) \&(X(x) \vee \mathfrak{A}(x)) \& \rightarrow X\left(b_{1}\right) \& \cdots\right. \\
\left.\& \neg X\left(b_{n}\right) \&(\neg X(x) \mathfrak{B}(x))\right],
\end{array}
$$

where $\mathfrak{A}(a)$ and $\mathfrak{B}(a)$ have no occurrences of any bound variables. Indeed, first order formulae are equivalent in our sense if they are semantically equivalent because of the completeness theorem. Consequently, some of the results in Chapter IV, [A], can be used here

${ }^{1}$ For information about the operation of substitution, refer to [C, §35.]

2 Among others, if $\mathbf{E q}(\mathfrak{A}, \mathfrak{B})$ then $\mathbf{E q}(\mathfrak{S}(\mathfrak{U}), \mathfrak{C}(\mathfrak{B})$ ) for all formulae $\mathfrak{A}, \mathfrak{B}$, $\mathfrak{C}(\mathfrak{A})$. 
and so, if the given formula is not in the form $\left({ }^{*}\right)$, we can find a formula equivalent to it which is. We will show the equivalence of $\left({ }^{*}\right)$ to the following:

$$
\prod_{i, j} a_{i} \neq b_{j} \& \forall x(\mathfrak{A}(x) \vee \mathfrak{B}(x)) \& \prod_{j} \mathfrak{A}\left(b_{j}\right) \& \prod_{i} \mathfrak{B}\left(a_{i}\right),
$$

where $\Pi$ is for conjunction of many terms.

It is easy to verify the pr-deducibility of $\left(^{*}\right) \rightarrow\left({ }^{* *}\right)$. To see the prdeducibility of $\left({ }^{* *}\right) \rightarrow\left(^{*}\right)$, first let $\mathfrak{S}(c)$ be $(\mathfrak{A}(c) \& \rightarrow \mathfrak{B}(c)) \bigvee c=a_{1}$ $\vee \ldots \vee c=a_{m}$, where $c$ is a new free variable, and let $(* * *)$ be the result of substitution of $\mathfrak{S}(c)$ for $X(c)$ in the scope of $\exists X$ in $\left(^{*}\right)$. Then it is not difficult to show the pr-deducibility if $\left({ }^{* *}\right) \rightarrow\left({ }^{* * *}\right)$ and of $(* * *) \rightarrow(*)$. This completes the proof.

Theorem I. A sequent of $U^{+}$is pr-deducible if [and only if] it is deducible.

Proof. We use induction on the number of impredicative applications of $\rightarrow \exists p$ and of $\rightarrow \exists s$ in the given deduction. Hence we may assume that there is only one such application and that its upper sequent is $\Gamma \rightarrow \Delta, \mathfrak{A}(\mathfrak{B})$ where $\mathfrak{B}$ is not of first order. The above lemma shows that there is a first order formula $\mathbb{E}$ such that $\mathfrak{A}(\mathfrak{B}) \rightarrow \mathfrak{A}(\mathfrak{E})$ is pr-deducible. By cut, we obtain $\Gamma \rightarrow \Delta, \mathfrak{A}(\mathfrak{E})$ predicatively. From this the original lower sequent can be obtained predicatively.

Theorem II. A sequent of $U$ is deducible without using the rule cut in $U$ if [and only if] it is deducible in $U^{+}$.

Proof. Suppose that a deduction in $U^{+}$is given. From the previous theorem, we may assume that this deduction is predicative. We define the grade of a formula to be the ordinal number $\omega \cdot m+n$ where $m$ is the number of occurrences of quantifiers on proposition or set variables and $n$ is the number of other logical connectives in the formula. By applying the Gentzen method of cut elimination as carried out in [G], and using the above definition of grade, we can transform the deduction in such a way that the grade of all cut formulae is 0 , and hence no application of cut was preceded by any logical rules of inference. In this deduction, we replace $a=b$ by $\forall X(X(a) \equiv X(b))$ everywhere. As is easily seen, the new axioms of $U^{+}$are pr-deducible in $U$ after this replacement. Thus, we obtain a deduction in $U$ of the given sequent such that the portions down to any applications of cut ${ }^{3}$ are predicative. By the Gentzen method, again, all these cuts can be

${ }^{3}$ Observe that applications of $\rightarrow \exists p$ and $\rightarrow \exists s$ in the lower part of the deduction may not be predicative. 
eliminated. Thus we have proved our theorem as well as the conjecture of $[T]$ for the unary second order language.

Remarks. As the referee suggested of the first version of this Note, the following are natural questions to be investigated:

(i) Does the cut elimination theorem hold in $U^{+}$, also?

(ii) Can predicativity be strengthened to the condition that $\mathfrak{B}$ in $\rightarrow \exists p$ and $\rightarrow \exists s$ are atomic formulae?

(iii) Are the deducible sequents of $U$ pr-deducible? The answers to these questions are all negative.

As to (i); Certainly $a=b \rightarrow b=a$ is deducible, for any variables $a$ and $b$. Assume that there were a deduction of this sequent without cut. Then the only rules available there would be those about structure. Hence all sequents in the deduction would be $a=b, \cdots, a=b$ $\rightarrow b=a, \cdots, b=a$. But no such sequent is an axiom. Thus, $a=b$ $\rightarrow b=a$. is not deducible without using cut.

As to (ii); Call the new condition sp (strong predicativity). First we notice that all sp-deducible sequents turn out to be "true" under the following "truth-value assignment": each atomic part receives "truth": propositional connectives are treated in the standard way: quantifiers are simply ignored; the "truth-value" of the sequent $\mathfrak{A}_{1}, \cdots, \mathfrak{A}_{m} \rightarrow \mathfrak{B}_{1}, \cdots, \mathfrak{B}_{n}$ is that of $\mathfrak{A}_{1} \& \cdots \& \mathfrak{A}_{m} \supset \mathfrak{B}_{1} \bigvee \cdots \vee \mathfrak{B}_{n}$. $\forall S(P \& S) \rightarrow \longrightarrow P$ is false in this assignment, and hence is not spdeducible. However, the above segment is pr-deducible, evidently!

As to (iii); We consider the following "model," R1: R1 is the ordered pair of the set of real numbers $\phi$, and of the Boolean set algebra generated by the half-open intervals $\Phi$. Remark that $\Phi$ is atomless. An "assignment" is a mapping of variables to elements of $\phi$, or of $\Phi$, or of the truth values, according to the species. Then semantical notions with respect to $\mathrm{Rl}$ can be defined, much in the standard way. We show that all pr-deducible sequents of $U$ are valid in Rl, by induction on the deductions. Every case but $\rightarrow \exists s$ is straightforward and easy to verify. So suppose that the upper sequent of $\rightarrow \exists s$ is valid in $\mathrm{Rl}$, and an assignment is given. Furthermore, we may assume that formulae in $\Gamma$ are true and those in $\Delta$ are false in the given assignment (hence, $\mathfrak{A}(\mathfrak{B})$ is true), for otherwise the verification is trivial. Then we are to show that $\exists X \mathfrak{A}(X)$ is also true; that is, there is an assignment which differs from the given one at $X()$ such that $\mathfrak{A}(X)$ is true. Suppose that $\mathfrak{A}(\mathfrak{B})$ is the result of substitution of a first order formula $\mathfrak{B}(x)$ for $X(x)$ in $\mathfrak{A}(X)$. Now we transform $\mathfrak{B}(x)$ in the following way: first restrict the scopes of negation signs to atomic parts by using de Morgan's law, then make the scopes of the quantifiers as small as possible by using disjunctive and conjunctive forms of matrices and 
renaming bound variables if necessary. The result is a combination, by means of $\&$ and $V$, of atomic formulae and formulae of the form $B(x), \exists y \subseteq(y)$ and $\forall y \mathfrak{D}(y)$ in which no bound variables occur except $x$ or $y$. Replace these formulae by the truth values under the given assignment, except those of the form $B(x)$. By computing truth values in regard to $\&$ and $V$, we obtain a Boolean combination of formulae $B_{1}(x), \cdots, B_{n}(x)$. Since the $B_{i}()$ are assigned to elements of $\Phi$, the above Boolean combination of these elements is an element of $\Phi$. In the new assignment, $X()$ is correlated to this element of $\Phi$. Then clearly $\mathfrak{A}(X)$ is true in this new assignment.

Finally, consider the sequent

$$
\rightarrow \exists X[X(a) \& \forall x[X(x) \supset \forall Y(Y(a) \equiv Y(x))]],
$$

which "says" that the singleton of $a$ exists. This sequent is not valid in Rl because $\Phi$ is atomless and for any two distinct real numbers there is a half-open interval containing the one and not the other. Hence $(\$)$ is not pr-deducible. However, it is deducible: the crucial step in the deduction is to "replace" $\forall Y(Y(a) \equiv Y(x))$ by $X(x)$.

\section{REFERENCES}

[A] W. Ackermann, Solvable cases of the decision problem, North-Holland, Amsterdam, 1954.

[C] A. Church, Introduction to mathematical logic, Princeton Univ. Press, Princeton, N. J., 1956.

[G] G. Gentzen, Untersuchungen über logische Schliessen, Math. Z., 39 (1953), 176-210, 405-431.

[T] G. Takeuti, On a generalized logic calculus, Japan. J. Math. 23 (1953), 39-96.

UNIVERSITÉ DE MONTREAL AND

NEW YORK UNIVERSITY 